TRANSACTIONS OF THE

AMERICAN MATHEMATICAL SOCIETY

Volume 363, Number 2, February 2011, Pages 681-697

S 0002-9947(2010)05146-3

Article electronically published on September 15, 2010

\title{
CONVERGENCE OF DIRICHLET POLYNOMIALS IN BANACH SPACES
}

\author{
ANDREAS DEFANT AND PABLO SEVILLA-PERIS
}

\begin{abstract}
Recent results on Dirichlet series $\sum_{n} a_{n} \frac{1}{n^{s}}, s \in \mathbb{C}$, with coefficients $a_{n}$ in an infinite dimensional Banach space $X$ show that the maximal width of uniform but not absolute convergence coincides for Dirichlet series and for $m$-homogeneous Dirichlet polynomials. But a classical non-trivial fact due to Bohnenblust and Hille shows that if $X$ is one dimensional, this maximal width heavily depends on the degree $m$ of the Dirichlet polynomials. We carefully analyze this phenomenon, in particular in the setting of $\ell_{p}$-spaces.
\end{abstract}

\section{INTRODUCTION}

Based on our results from [13, we continue the recent study of ordinary Dirichlet series from [11. Let $X$ be some Banach space and $m \in \mathbb{N}$. We call a series

$$
\sum_{n} a_{n} \frac{1}{n^{s}}, s \in \mathbb{C},
$$

a Dirichlet series in $X$ if all its coefficients $a_{n}$ belong to $X$. We call it an $m$ homogeneous Dirichlet polynomial in $X$ whenever $a_{n}=0$ for all indices $n$ which do not have precisely $m$ prime divisors (counted according to their multiplicity). Here the Banach space $\ell_{p}$ of all absolutely $p$-summable scalar sequences will be of special interest. As in the case of Dirichlet series with complex coefficients, each vector valued Dirichlet series has associated with it an abscissa of uniform convergence and an abscissa of absolute convergence. More precisely, let

$$
\sigma_{a}=\sigma_{a}^{X}=\sigma_{a}\left(\sum_{n} a_{n} \frac{1}{n^{s}}\right):=\inf r \text { and } \sigma_{u}=\sigma_{u}^{X}=\sigma_{u}\left(\sum_{n} a_{n} \frac{1}{n^{s}}\right):=\inf r
$$

be the infimum taken over all $r$ such that on the half plane $[\operatorname{Re} s \geq r]$ the series converges absolutely and uniformly, respectively. In other terms, $\left[\operatorname{Re} s \geq \sigma_{a}\right]$ is the largest half plane such that $\sum_{n} a_{n} \frac{1}{n^{s}}$ converges absolutely in $\left[\operatorname{Re} s \geq \sigma_{a}+\varepsilon\right]$ for each $\varepsilon>0$, and $\left[\operatorname{Re} s \geq \sigma_{a}\right]$ is the largest half plane for which the series for every $\varepsilon$ converges uniformly in $\left[\operatorname{Re} s \geq \sigma_{u}+\varepsilon\right]$. Define

$$
T_{m}(p, q)=\sup \sigma_{a}^{\ell_{q}}-\sigma_{u}^{\ell_{p}},
$$

Received by the editors July 14, 2008 and, in revised form, March 7, 2009.

2000 Mathematics Subject Classification. Primary 46B07; Secondary 32A05, 46B09, 46G20, 30B50.

Key words and phrases. Vector valued Dirichlet series, Dirichlet polynomials, Banach spaces.

Both authors were supported by the MEC Project MTM2008-03211. The second author was partially supported by grants PR2007-0384 (MEC) and UPV-PAID-00-07.

(C)2010 American Mathematical Society Reverts to public domain 28 years from publication 
where the supremum is taken over all possible $m$-homogeneous Dirichlet polynomials in $\ell_{p}$. Clearly, $T_{m}(p, q)$ determines the width of the largest possible strip where an $m$-homogeneous Dirichlet polynomial $\sum_{n} a_{n} \frac{1}{n^{s}}$ in $\ell_{p}$ converges uniformly but does not converge absolutely, considered as a series in $\ell_{q}$. The main result of this paper is the following:

Theorem 1.1. For each $m$ and $1 \leq p \leq q \leq \infty$

$$
T_{m}(p, q)= \begin{cases}\frac{m-2(1 / p-\max \{1 / q, 1 / 2\})}{2 m} & \text { if } 1 \leq p \leq 2, \\ 1-\frac{1}{p} & \text { if } 2 \leq p .\end{cases}
$$

Moreover, for every $0 \leq \sigma \leq T_{m}(p, q)$ there exists an m-homogeneous Dirichlet polynomial in $\ell_{p}$ for which $\sigma_{a}^{\overline{\ell_{q}}}-\sigma_{u}^{\ell_{p}}=\sigma$.

The theory of Dirichlet series plays an important role in analytic number theory (see e.g. Apostol's books [1, 2]), and results of the preceding type have a long history. Bohr in [5, 6] defined the number $T$, the supremum of all width $\sigma_{a}-\sigma_{u}$ taken over all scalar Dirichlet series, and proved that $T \leq 1 / 2$. Eighteen years later Bohnenblust and Hille [4] showed that in fact

$$
T=\frac{1}{2}
$$

(see the beautiful survey 3 of Boas and also 17]). They implicitly defined the number $T_{m}$, replacing in the definition of $T$ all scalar Dirichlet series by all scalar $m$-homogeneous Dirichlet polynomials, and proved that

$$
T_{m}=\frac{m-1}{2 m} .
$$

Here $T_{m}$ denotes the supremum of all width $\sigma_{a}-\sigma_{u}$ with respect to all $m$-homogeneous Dirichlet polynomials in $\mathbb{C}$. Then letting $m$ tend to $\infty$ leads to (2), since $T_{m} \leq T$ for all $m$. For another deep study of the Bohr-Bohnenblust-Hille equalities from (21) and (3) see also [18 and 22. Although our techniques are very different from those of Bohnenblust and Hille in the scalar case, an easy analysis of our proof of Theorem 1.1 (see Section 4.1) also shows that the Dirichlet polynomial attaining the maximal width can even be chosen to have complex coefficients if and only if $p=1$ and $2 \leq q \leq \infty$.

More recently, in [11] a similar question for Dirichlet series $\sum_{n} a_{n} \frac{1}{n^{s}}, s \in \mathbb{C}$, with coefficients $a_{n}$ in a fixed Banach space $X$ is addressed. Clearly, the definition of the maximal width $T(X)$ and $T_{m}(X)$ then again follows the same lines, but considerably different phenomena occur. It turns out that still $T(X)=1 / 2$ and $T_{m}(X)=\frac{m-1}{2 m}$ whenever $X$ is finite dimensional. However, if $X$ is infinite dimensional, then both numbers coincide and depend only on the geometry of the underlying Banach space $X$. More precisely, the main result from [11] states that for an infinite dimensional Banach space $X$ and for each $m$ we have $T(X)=T_{m}(X)=1-1 / \cot (X)$, where $\cot (X)$ is the optimal cotype of $X$ (see below for definitions). For special Banach spaces this optimal cotype is computable which e.g. gives

$$
T\left(\ell_{p}\right)=T_{m}\left(\ell_{p}\right)= \begin{cases}1 / 2 & \text { if } 1 \leq p \leq 2, \\ 1-1 / p & \text { if } 2 \leq p \leq \infty\end{cases}
$$


This means that in infinite dimensions Bohr's strips do not distinguish between arbitrary Dirichlet series and $m$-homogeneous Dirichlet polynomials. A careful study of this phenomenon for $\ell_{p}$-spaces led to Theorem [1.1 which shows how to 'find the polynomials back' in our more general setting. Moreover, this result still covers (3) (take $p=1$ and $q=2$, and consider scalar series only) and (4) (take $p=q$ ) as special cases.

Using results and techniques of Bohr [5, 6], Bohnenblust-Hille [4] and [11, 13, the proof will be given in Sections 3 , 4 and 5 .

\section{Preliminaries}

In all what follows, capital letters such as $X, Y$ will denote Banach spaces over $\mathbb{C}$, whose duals will be denoted $X^{*}, Y^{*}, \ldots$. By a Banach sequence space we will mean a Banach space $X \subseteq \mathbb{C}^{\mathbb{N}}$ of sequences in $\mathbb{C}$ satisfying $\ell_{1} \subseteq X \subseteq \ell_{\infty}$, and if $x \in \mathbb{C}^{\mathbb{N}}$ and $y \in X$ with $|x| \leq|y|$, then $x \in X$ and $\|x\| \leq\|y\|$. For each $n \in \mathbb{N}$ we denote $X_{n}=\operatorname{span}\left\{e_{1}, \ldots, e_{n}\right\}$ in $X$. Examples of such Banach sequence spaces are the $\ell_{p}$-spaces whose norm will be denoted $\|\cdot\|_{p} ; c_{0}$ as usual stands for the Banach space of all zero sequences. The conjugate of a real number $\gamma>1$ is the number $\gamma^{\prime}$ such that $\frac{1}{\gamma}+\frac{1}{\gamma^{\prime}}=1$; if $\gamma=1$, then $\gamma^{\prime}=\infty$ and we use the convention $\frac{1}{\infty}=0$. We will denote by $\mathbb{N}_{0}^{(\mathbb{N})}$ the set of all sequences of natural numbers (multi-indices) whose entries are all zero except for a finite number; the length of such a multi-index $\alpha$ is defined as the biggest $k$ such that $\alpha_{k} \neq 0$. Throughout the paper $\left(p_{k}\right)_{k}$ denotes the sequence of prime numbers. If $\alpha=\left(\alpha_{k}\right)_{k}$ is a multi-index in $\mathbb{N}_{0}^{(\mathbb{N})}$ of length $k$, then we write $p^{\alpha}=p_{1}^{\alpha_{1}} p_{2}^{\alpha_{2}} \cdots p_{k}^{\alpha_{k}}$ and $\alpha !=\alpha_{1} ! \cdots \alpha_{k}$ !. For any natural number $n$ with prime decomposition $n=p_{1}^{\alpha_{1}} p_{2}^{\alpha_{2}} \cdots p_{k}^{\alpha_{k}}$, following standard notation we write $\Omega(n)=\alpha_{1}+\alpha_{2}+\cdots+\alpha_{k}$. As a consequence, the symbol $p$ will have along the paper two different meanings: either the sequence of primes or the index of an $\ell_{p}$-space.

The Banach space of all (bounded and linear) operators between two Banach spaces $X$ and $Y$ is denoted by $\mathcal{L}(X, Y)$, and the Banach space of all (bounded) $m$-linear mappings from $X \times \cdots \times X$ to $Y$ is denoted by $\mathcal{L}\left({ }^{m} X, Y\right)$. With the norm

$$
\|A\|=\sup \left\{\left\|A\left(x_{1}, \ldots, x_{m}\right)\right\|_{Y}:\left\|x_{i}\right\|_{X} \leq 1, i=1, \ldots, m\right\}
$$

this is a Banach space. A continuous mapping between Banach spaces $P: X \rightarrow Y$ is called an $m$-homogeneous polynomial if there exists a continuous $m$-linear mapping $A$ from $X$ to $Y$ such that $P(x)=A(x, \ldots, x)$ for every $x \in X$. The space of $m$ homogeneous polynomials between $X$ and $Y$ is denoted by $\mathcal{P}\left({ }^{m} X, Y\right)$, and with the norm $\|P\|=\sup _{\|x\| \leq 1}\|P(x)\|$ it is a Banach space. If $Y=\mathbb{C}$, then $\mathcal{P}\left({ }^{m} X, \mathbb{C}\right)$ is simply denoted by $\mathcal{P}\left({ }^{m} X\right)$. We will make use of the fact that if $X$ is a finite dimensional Banach space, then we have the following identifications:

$$
\mathcal{P}\left({ }^{m} X, Y\right)=\mathcal{P}\left({ }^{m} X\right) \otimes_{\varepsilon} Y=\bigotimes_{\varepsilon_{s}}^{m, s} X^{*} \otimes_{\varepsilon} Y
$$

by identifying each element $x^{*} \otimes \cdots \otimes x^{*} \otimes y$ with the polynomial $x \rightsquigarrow x^{*}(x)^{m} y$. As usual the projective and injective tensor norms are denoted by $\pi$ and $\varepsilon$, respectively, and the symmetric projective and injective tensor norms are denoted by $\pi_{s}$ and $\varepsilon_{s}$. Detailed studies of the theory of polynomials, tensor products and symmetric tensor products can be found in [9, 15, 16. If $X$ is a Banach sequence space, then every 
$P \in \mathcal{P}\left({ }^{m} X, Y\right)$ has a monomial series expansion

$$
\sum_{\alpha \in \mathbb{N}_{0}^{(\mathbb{N})}} c_{\alpha}(P) x^{\alpha}
$$

where as usual $x^{\alpha}=x_{1}^{\alpha_{1}} x_{2}^{\alpha_{2}} \cdots$. If $\alpha$ is a multi-index of length $n$, then the coefficient $c_{\alpha}(P)=c_{\alpha}$ is simply defined through the monomial expansion of the restriction of the polynomial to $X_{n}=\operatorname{span}\left\{e_{1}, \ldots, e_{n}\right\}$ (which clearly can be identified with $\mathbb{C}^{n}$ ). It is not in general true that (6) converges pointwise to $P$. A deep and complete study of when this happens in the scalar-valued case can be found in 12.

Following [20, Def. 1.e.12]), a Banach space $X$ is said to have cotype $q$ (with $2 \leq q<\infty$ ) whenever there is some constant $C>0$ such that for each choice of finitely many vectors $x_{1}, \ldots, x_{n} \in X$ we have

$$
\left(\sum_{i=1}^{n}\left\|x_{i}\right\|^{q}\right)^{1 / q} \leq C\left(\int_{0}^{1}\left\|\sum_{i=1}^{n} r_{i}(t) x_{i}\right\|^{2} d t\right)^{1 / 2},
$$

where $r_{i}$ stands for the $i$-th Rademacher function on $[0,1]$. As usual, the best such $C$ is denoted by $\mathrm{C}_{q}(X)$. It is well known that $\ell_{p}$ has cotype $\max \{p, 2\}$. By $\cot (X)$ we denote the infimum over all possible $q$ such that $X$ has cotype $q$.

\section{The proof: Part I. The upper bound}

We begin the proof of Theorem 1.1 by obtaining the upper bound for $T_{m}(p, q)$. In the classical theory of Dirichlet series the corresponding abscissas of convergence of a given Dirichlet series can be computed by means of the Hadamard Formulas (see e.g. 2, Chapter 8], [22, (I.4)]). A straightforward modification of the proofs gives the following vector valued version of the Hadamard Formulas for a Dirichlet series $\sum_{n} a_{n} \frac{1}{n^{s}}$ with coefficients in a Banach space $X$, provided $\sum_{n} a_{n}$ is divergent:

$$
\begin{gathered}
\sigma_{a}=\limsup _{N \rightarrow \infty} \frac{\log \left(\sum_{n=1}^{N}\left\|a_{n}\right\|_{X}\right)}{\log N}, \\
\sigma_{u}=\limsup _{N \rightarrow \infty} \frac{\log \left(\sup _{t \in \mathbb{R}}\left\|\sum_{n=1}^{N} a_{n} n^{i t}\right\|_{X}\right)}{\log N} .
\end{gathered}
$$

One of Bohr's main contributions was to relate Dirichlet series with power series in infinitely many variables. This relation can nowadays be treated from the point of view of holomorphic functions on infinite dimensional Banach spaces, and carries to vector valued Dirichlet series, in the so-called 'Bohr's trick' (see [11, Lemma 5]): If $a_{1}, \ldots, a_{N} \in X$ and we write $c_{\alpha}=a_{p^{\alpha}}$, then

$$
\sup _{t \in \mathbb{R}}\left\|\sum_{n=1}^{N} a_{n} n^{i t}\right\|_{X}=\sup _{x \in B_{\ell N}^{N}}\left\|\sum_{\alpha \in \Lambda_{N}} c_{\alpha} x^{\alpha}\right\|_{X},
$$

where $\Lambda_{N}=\left\{\alpha \in \mathbb{N}_{0}^{(\mathbb{N})}: 1 \leq p^{\alpha} \leq N\right\}$. Let us point out that if $a_{1}, \ldots, a_{N}$ are so that $a_{n}=0$ whenever $\Omega(n) \neq m$, what we have on the left-hand side of (9) is an $m$-homogeneous Dirichlet polynomial. On the right-hand side we have an 
$m$-homogeneous polynomial; therefore

$$
\sup _{x \in B_{\ell_{\infty}^{N}}}\left\|\sum_{\alpha \in \Lambda_{N}} c_{\alpha} x^{\alpha}\right\|_{X}=\left\|\sum_{\alpha \in \Lambda_{N}} c_{\alpha} x^{\alpha}\right\|_{\mathcal{P}\left({ }^{m} c_{0}, X\right)} .
$$

This suggests that there must be a close relation between the uniform convergence of Dirichlet polynomials in $X$ and polynomials on $c_{0}$ with values in $X$. More precisely, for every $m$-homogeneous Dirichlet polynomial $\sum_{\Omega(n)=m} a_{n} / n^{s}$ we have

$$
\sigma_{u}=\inf \left\{\mu \in \mathbb{R}: \text { there exists } P \in \mathcal{P}\left({ }^{m} c_{0}, X\right), c_{\alpha}(P)=\frac{a_{p^{\alpha}}}{p^{\mu \alpha}}\right\} .
$$

This follows from an analogous formula [11, Corollary 2] for general Dirichlet series where the polynomials $P$ have to be replaced by holomorphic functions and from the fact that if all coefficients of a holomorphic function with $|\alpha| \neq m$ vanish, then it is an $m$-homogeneous polynomial.

We can now prove the upper bound in Theorem 1.1. Let us consider

$$
r= \begin{cases}\frac{2 m}{m+2\left(\frac{1}{p}-\max \left\{\frac{1}{q}, \frac{1}{2}\right\}\right)} & \text { if } p \leq 2, \\ p & \text { if } p \geq 2 .\end{cases}
$$

By the main result in [13] (Theorem 4) there exists $c>0$ such that

$$
\left(\sum_{\alpha}\left\|c_{\alpha}(P)\right\|_{q}^{r}\right)^{1 / r} \leq c\|P\|_{\mathcal{P}\left({ }^{m} c_{0}, \ell_{p}\right)}
$$

for every $P \in \mathcal{P}\left({ }^{m} c_{0}, \ell_{p}\right)$. Then if $a_{1}, \ldots, a_{N} \in \ell_{p}$ are all 0 whenever $\Omega(n) \neq m$ and if we denote $\Lambda_{N, m}=\left\{\alpha \in \Lambda_{N}:|\alpha|=m\right\}$, we can apply (11), Hölder's inequality and (9) to get

$$
\begin{aligned}
& \sum_{n=1}^{N}\left\|a_{n}\right\|_{q} \leq N^{1 / r^{\prime}}\left(\sum_{n=1}^{N}\left\|a_{n}\right\|_{q}^{r}\right)^{1 / r}=N^{1 / r^{\prime}}\left(\sum_{\alpha \in \Lambda_{N, m}}\left\|c_{\alpha}\right\|_{q}^{r}\right)^{1 / r} \\
& \leq c N^{1 / r^{\prime}}\left\|\sum_{\alpha \in \Lambda_{N, m}} c_{\alpha} z^{\alpha}\right\|_{\mathcal{P}\left({ }^{m} c_{0}, \ell_{p}\right)}=c N^{1 / r^{\prime}} \sup _{t \in \mathbb{R}}\left\|\sum_{n=1}^{N} a_{n} n^{i t}\right\|_{p} .
\end{aligned}
$$

By (7) and (8) we have for every $m$-homogeneous Dirichlet polynomial such that $\sum_{n} a_{n}$ is divergent in $\ell_{q}$,

$$
\sigma_{a}^{\ell_{q}}\left(\sum_{n} a_{n} \frac{1}{n^{s}}\right) \leq \frac{1}{r^{\prime}}+\sigma_{u}^{\ell_{p}}\left(\sum_{n} a_{n} \frac{1}{n^{s}}\right) .
$$

If $\sum_{n} a_{n}$ is convergent in $\ell_{q}$ we consider two different cases. On one hand if $\sum a_{n} / n^{s}$ is convergent in $\ell_{q}$ for every $s$, then it is easily proved that it also converges absolutely for every $s$; in other words, $\sigma_{a}^{\ell_{q}}\left(\sum a_{n} / n^{s}\right)=-\infty$. Then (13) trivially holds. On the other hand, if $\sum a_{n} / n^{s_{0}}$ diverges in $\ell_{q}$ for some $s_{0}$, then it also diverges in $\ell_{p}$. Then let $b_{n}=a_{n} / n^{s_{0}}$, and we have

$$
\sigma_{a}^{\ell_{q}}\left(\sum_{n} b_{n} \frac{1}{n^{s}}\right) \leq \frac{1}{r^{\prime}}+\sigma_{u}^{\ell_{p}}\left(\sum_{n} b_{n} \frac{1}{n^{s}}\right)
$$

But by the definition, $\sigma_{a}^{\ell_{q}}\left(\sum_{n} b_{n} / n^{s}\right)=\sigma_{a}^{\ell_{q}}\left(\sum_{n} a_{n} / n^{s}\right)+s_{0}$, and similarly for $\sigma_{u}^{\ell_{p}}$. This finally gives that (13) holds for every $m$-homogeneous Dirichlet polynomial 
and implies

$$
T_{m}(p, q) \leq \begin{cases}\frac{m-2(1 / p-\max \{1 / q, 1 / 2\})}{2 m} & \text { if } 1 \leq p \leq 2, \\ \frac{1}{p^{\prime}} & \text { if } 2 \leq p .\end{cases}
$$

\section{The Proof: Part II. The Lower Bound}

Since $T_{m}(p, q)$ is defined as a supremum, it is clear that if in each case we produce an $m$-homogeneous Dirichlet polynomial for which $\sigma_{a}^{\ell_{q}}-\sigma_{u}^{\ell_{p}}$ is exactly the number in (11), we will have the desired lower bound for $T_{m}(p, q)$. To do that we need the following technical result. We now adopt a general approach, using operators between Banach spaces. Later we will apply Proposition 4.1 to the inclusion $v=$ id $: \ell_{p} \hookrightarrow \ell_{q}$; also by taking $v=\operatorname{id}_{\mathbb{C}}$ we will recover the results in Sections 4 and 5 of [4].

Proposition 4.1. Let $v: X \rightarrow Y$ be a non-zero linear operator. Let us fix $\rho \in \mathbb{N}$, $\rho>1$ and $m \in \mathbb{N}$. If there exist $\gamma, \beta$ with $m \geq \gamma-\beta>0$ and constants $K, C>0$ so that for each $n$ there exists $P_{n} \in \mathcal{P}\left({ }^{m} \ell_{\infty}^{\rho^{n}}, X\right)$ with $\left\|P_{n}\right\| \leq C\left(\rho^{n}\right)^{\gamma}$ and $\inf _{|\alpha|=m}\left\|c_{\alpha}\left(v P_{n}\right)\right\| \geq K\left(\rho^{n}\right)^{\beta}$, then there exists an $m$-homogeneous Dirichlet polynomial in $X$ for which $\sigma_{a}^{Y}\left(\sum v a_{n} / n^{s}\right)-\sigma_{u}^{X}\left(\sum a_{n} / n^{s}\right) \geq 1 / q^{\prime}$, where $q=$ $m /(\gamma-\beta)$.

Proof. Let us note first that if $m=\gamma-\beta$ the statement is trivial since in this case $1 / q^{\prime}=0$, and we are simply saying that there exists some Dirichlet polynomial for which $\sigma_{a}^{Y}-\sigma_{u}^{X} \geq 0$. We can then assume $m>\gamma-\beta$; first of all we divide each $x \in c_{0}$ into blocks $x_{1}, x_{2}, \ldots$ so that the block $x_{n}$ has length $\rho^{n}$,

$$
x=\left(x_{1}(1), \ldots, x_{1}(\rho), x_{2}(1), \ldots, x_{2}\left(\rho^{2}\right), x_{3}(1), \ldots, x_{3}\left(\rho^{3}\right), \ldots\right),
$$

and define the following polynomial blockwise:

$$
P(x)=\sum_{n=1}^{\infty} \frac{1}{n^{2}}\left(\rho^{n}\right)^{-\gamma} P_{n}\left(x_{n}\right) .
$$

Clearly by our assumption on $\left\|P_{n}\right\|$ we have $P \in \mathcal{P}\left({ }^{m} c_{0}, X\right)$. Now let $\sum_{\Omega(n)=m} a_{n} \frac{1}{n^{s}}$ be the Dirichlet polynomial in $X$ associated to $P$ (i.e. $a_{p^{\alpha}}=c_{\alpha}$ ). By (10) this satisfies $\sigma_{u} \leq 0$; let us now show that for every fixed $0<\delta<1$ we have $\sigma_{a}\left(\sum v a_{n} / n^{s}\right) \geq \frac{1}{q^{\prime}} \frac{1-\delta}{1+\delta}$. This will give $\sigma_{a}\left(\sum v a_{n} / n^{s}\right) \geq 1 / q^{\prime}$ and complete the proof. Given $0<\delta<1$ we choose $b<1$ so that $b^{1-\delta} \rho^{\delta}>1$. We define $x=(x(k))_{k}$ blockwise by $x_{n}(k)=\left(\frac{b}{\rho}\right)^{n(1-\delta) / q^{\prime}}$. Let us check that $x \in \ell_{q^{\prime} /(1-\delta)}$; indeed

$$
\begin{aligned}
& \sum_{k=1}^{\infty} x(k)^{q^{\prime} /(1-\delta)}=\sum_{n=1}^{\infty} \sum_{k=1}^{\rho^{n}} x_{n}(k)^{q^{\prime} /(1-\delta)} \\
&=\sum_{n=1}^{\infty} \sum_{k=1}^{\rho^{n}}\left(\frac{b}{\rho}\right)^{n \frac{1-\delta}{q^{\prime}} \frac{q^{\prime}}{1-\delta}}=\sum_{n=1}^{\infty} b^{n}=\frac{b}{1-b}<\infty .
\end{aligned}
$$


On the other hand, by our assumption on the lower bound for $c_{\alpha}\left(v P_{n}\right)$,

$$
\begin{aligned}
\sum_{|\alpha|=m} \| & c_{\alpha}(v P) x^{\alpha}\left\|=\sum_{n=1}^{\infty} \sum_{|\alpha|=m} \frac{1}{n^{2}}\left(\rho^{n}\right)^{-\gamma}\right\| c_{\alpha}\left(v P_{n}\right) x_{n}^{\alpha} \| \\
& =\sum_{n=1}^{\infty} \frac{1}{n^{2}}\left(\rho^{n}\right)^{-\gamma} \sum_{|\alpha|=m}\left\|c_{\alpha}\left(v P_{n}\right)\right\|\left|x_{n}\right|^{\alpha} \\
& \geq K \sum_{n=1}^{\infty} \frac{1}{n^{2}}\left(\rho^{n}\right)^{-\gamma}\left(\rho^{n}\right)^{\beta} \sum_{|\alpha|=m}\left|x_{n}\right|^{\alpha} \\
& =K \sum_{n=1}^{\infty} \frac{1}{n^{2}}\left(\rho^{n}\right)^{-(\gamma-\beta)}\left(\sum_{k=1}^{\rho^{n}}\left(\frac{b}{\rho}\right)^{n(1-\delta) / q^{\prime}}\right)^{m} \\
& =K \sum_{n=1}^{\infty} \frac{1}{n^{2}}\left(\rho^{n}\right)^{-m / q}\left(\frac{b}{\rho}\right)^{m n(1-\delta) / q^{\prime}}\left(\rho^{n}\right)^{m} \\
& =K \sum_{n=1}^{\infty} \frac{1}{n^{2}}\left(\left(b^{1-\delta} \rho^{\delta}\right)^{m / q^{\prime}}\right)^{n} .
\end{aligned}
$$

The last term is $\infty$ since $b^{1-\delta} \rho^{\delta}>1$.

Since $x \in \ell_{\frac{q^{\prime}}{1-\delta}}$ and is decreasing, we have for every $n$

$$
x_{n} \leq \frac{b}{1-b} \frac{1}{n^{\frac{1-\delta}{q^{\prime}}}} .
$$

On the other hand, by the prime number theorem, $p_{n} \leq D_{1} n \log n \leq D_{2} n^{1+\delta}$ for some constants $D_{1}, D_{2}$. Hence there is some $D_{3}$ such that

$$
x_{n} \leq \frac{b}{1-b} \frac{1}{n^{\frac{1-\delta}{q^{\prime}}}} \leq D_{3} \frac{b}{1-b} \frac{1}{p_{n}^{\frac{1}{1+\delta} \frac{1-\delta}{q^{\prime}}}} .
$$

Then for the Dirichlet polynomial $\sum_{\Omega(n)=m} v a_{n} \frac{1}{n^{s}}$ the following holds:

$$
\sum_{\Omega(n)=m}\left\|v a_{n}\right\| \frac{1}{n^{\frac{1}{q^{\prime}} \frac{1-\delta}{1+\delta}}}=\sum_{|\alpha|=m}\left\|v a_{p^{\alpha}}\right\| \frac{1}{\left(p^{\alpha}\right)^{\frac{1}{q^{\prime}} \frac{1-\delta}{1+\delta}}} \geq D_{3}^{-1} \frac{1-b}{b} \sum_{|\alpha|=m}\left\|c_{\alpha}(v P)\right\|\left|x^{\alpha}\right| .
$$

We know that the last term does not converge; this implies $\sigma_{a}\left(\sum v a_{n} / n^{s}\right) \geq \frac{1}{q^{\prime}} \frac{1-\delta}{1+\delta}$.

Taking $v=$ id $: \ell_{p} \hookrightarrow \ell_{q}$ we have that in order to prove the remaining inequality in (1), it is enough to find in each case sequences of polynomials $P_{n} \in$ $\mathcal{P}\left({ }^{m} \ell_{\infty}^{\rho^{n}}, \ell_{p}\right)$ satisfying the conditions in Proposition 4.1. Let us point out that if $P \in \mathcal{P}\left({ }^{m} c_{0}, \ell_{p}\right)$, then $v P \in \mathcal{P}\left({ }^{m} c_{0}, \ell_{q}\right)$. Then the conditions in Proposition 4.1 read as $\left\|P_{n}\right\|_{\mathcal{P}\left(m \ell_{\infty}^{\left.\rho^{n}, \ell_{p}\right)}\right.} \leq C\left(\rho^{n}\right)^{\gamma}$ and $\inf _{|\alpha|=m}\left\|c_{\alpha}\left(P_{n}\right)\right\|_{q} \geq K\left(\rho^{n}\right)^{\beta}$. We consider three different cases and treat each one separately.

4.1. The case $1 \leq p \leq q \leq 2$. In this case we need the following version of Chevét's inequalities (see [23, (43.2)] for the bilinear version and [8, Lemma 6] for the $m$-linear version) that can be viewed as some kind of vector valued version of [10, Corollary 3.2]. 
Lemma 4.2. Let $E=\left(\mathbb{C}^{n},\|\|_{E}\right)$ and $F=\left(\mathbb{C}^{N},\|\|_{F}\right)$. Then there exists a constant $K>0$ depending only on $m$ such that for every choice of scalars $\left(\lambda_{\alpha}\right)_{|\alpha|=m}$ we have

$$
\begin{aligned}
& \int\left\|\sum_{|\alpha|=m} \lambda_{\alpha}\left(\sum_{k=1}^{N} g_{\alpha, k}(\omega) e_{k}\right) x^{\alpha}\right\|_{\mathcal{P}\left({ }^{m} E, F\right)} d \omega \\
& \leq K \sup _{|\alpha|=m}\left(\left|\lambda_{\alpha}\right| \sqrt{\frac{\alpha !}{m !}}\right) \\
& \quad\left[\| \text { id }: E \rightarrow \ell_{2}^{n}\left\|^{m-1}\right\| \text { id }: \ell_{2}^{N} \rightarrow F\left\|\int\right\| \sum_{j=1}^{n} g_{j}(\omega) e_{j} \|_{E^{\prime}} d \omega\right. \\
&\left.+\| \text { id }: E \rightarrow \ell_{2}^{n}\left\|^{m} \int\right\| \sum_{j=1}^{N} g_{j}(\omega) e_{j} \|_{F} d \omega\right],
\end{aligned}
$$

where $\left(g_{j}\right)_{j}$ and $\left(g_{\alpha, k}\right)_{\alpha, k}$ are families of independent standard Gaussian random variables.

Proof. Let us choose independent Gaussian random variables $g_{\alpha, k}, g_{\alpha}$ and $g_{j}$ for $|\alpha|=m, k=1, \ldots, N$ and $j=1, \ldots, N$. By Chevét's inequality [23, (46.3)] and (5) we have

$$
\begin{aligned}
\int\left\|\sum_{\alpha, k} \lambda_{\alpha} g_{\alpha, k}(\omega) x^{\alpha} \otimes e_{k}\right\|_{\mathcal{P}\left({ }^{m} E\right) \otimes_{\varepsilon} F} d \omega & \\
\leq & c\left(\int\left\|\sum_{|\alpha|=m} \lambda_{\alpha} g_{\alpha}(\omega) x^{\alpha}\right\|_{\mathcal{P}\left({ }^{m} E\right)} d \omega \| \text { id }: \ell_{2}^{N} \longrightarrow F \|\right. \\
& \left.\quad+\sup _{\gamma \in B_{\mathcal{P}\left(m_{E}\right)^{\prime}}}\left(\sum_{|\alpha|=m}\left|\left\langle\lambda_{\alpha} x^{\alpha}, \gamma\right\rangle\right|^{2}\right)^{1 / 2} \int\left\|\sum_{j=1}^{N} g_{j}(\omega) e_{j}\right\|_{F} d \omega\right) .
\end{aligned}
$$

By [10, Corollary 3.2] there exists some constant $K>0$ such that

$$
\begin{aligned}
& \left.\int\left\|\sum_{|\alpha|=m} \lambda_{\alpha} g_{\alpha}(\omega) x^{\alpha}\right\|_{\mathcal{P}(m} E\right) \\
& \leq K=\int \sup _{x \in B_{E}}\left|\sum_{|\alpha|=m} \lambda_{\alpha} g_{\alpha}(\omega) x^{\alpha}\right| d \omega \\
& \quad \leq K|\alpha|=m \\
& \sup _{|\alpha|=m}\left(\left|\lambda_{\alpha}\right| \sqrt{\frac{\alpha !}{m !}}\right)\left(\sup _{x \in B_{E}}\left(\sum_{k=1}^{n}\left|x_{k}\right|^{2}\right)^{1 / 2}\right)^{m-1} \int \sup _{x \in B_{E}}\left|\sum_{i=1}^{n} g_{i}(\omega) x_{i}\right| d \omega .
\end{aligned}
$$

Here we have $\left(\sup _{x \in B_{E}}\left(\sum_{k=1}^{n}\left|x_{k}\right|^{2}\right)^{1 / 2}\right)^{m-1}=\|$ id $: E \longrightarrow \ell_{2}^{n} \|^{m-1}$. For the factor still to be estimated we do the usual identification $\mathcal{P}\left({ }^{m} E\right)=\otimes_{\varepsilon_{s}}^{m, s} E^{*}$; for each $m$-homogeneous polynomial we have three different possible representations

$$
\sum_{i_{1}, \ldots, i_{m}} \lambda_{i_{1}, \ldots, i_{m}} e_{i_{1}} \otimes \cdots \otimes e_{i_{m}}=\sum_{i_{1}, \ldots, i_{m}} \lambda_{i_{1}, \ldots, i_{m}} x_{i_{1}} \cdots x_{i_{m}}=\sum_{|\alpha|=m} \frac{m !}{\alpha !} \lambda_{\alpha} x^{\alpha} .
$$


Then

$$
\begin{aligned}
& \sup _{\substack{\gamma \in \mathcal{P}(m E)^{*} \\
\|\gamma\| \| 1}}\left(\sum_{|\alpha|=m}\left|\left\langle\lambda_{\alpha} x^{\alpha}, \gamma\right\rangle\right|^{2}\right)^{1 / 2}=\sup _{\substack{\gamma \in \mathcal{P}(m E)^{*} \\
\|\gamma\| \leq 1}}\left(\sum_{|\alpha|=m} \frac{\alpha !}{m !} \frac{m !}{\alpha !}\left|\left\langle\lambda_{\alpha} x^{\alpha}, \gamma\right\rangle\right|^{2}\right)^{1 / 2} \\
& \leq \sup _{|\alpha|=m}\left(\left|\lambda_{\alpha}\right| \sqrt{\frac{\alpha !}{m !}}\right) \sup _{\substack{\gamma \in \mathcal{P}(m E)^{*} \\
\|\gamma\| \leq 1}}\left(\sum_{|\alpha|=m} \frac{m !}{\alpha !}\left|\left\langle x^{\alpha}, \gamma\right\rangle\right|^{2}\right)^{1 / 2} \\
& =\sup _{|\alpha|=m}\left(\left|\lambda_{\alpha}\right| \sqrt{\frac{\alpha !}{m !}}\right) \sup _{\substack{\gamma \in \mathcal{P}(m E)^{*} \\
\|\gamma\| \leq 1}}\left(\sum_{i_{1}, \ldots, i_{m}}\left|\left\langle x_{i_{1}} \cdots x_{i_{m}}, \gamma\right\rangle\right|^{2}\right)^{1 / 2} \\
& \leq \sup _{|\alpha|=m}\left(\left|\lambda_{\alpha}\right| \sqrt{\frac{\alpha !}{m !}}\right) \sup _{\substack{\gamma \in\left(\otimes_{m}^{m} E^{*}\right)^{*} \\
\|\gamma\| \leq 1}}\left(\sum_{i_{1}, \ldots, i_{m}}\left|\left\langle e_{i_{1}} \otimes \cdots \otimes e_{i_{m}}, \gamma\right\rangle\right|^{2}\right)^{1 / 2} \\
& =\sup _{|\alpha|=m}\left(\left|\lambda_{\alpha}\right| \sqrt{\frac{\alpha !}{m !}}\right) \sup _{\substack{x \in \otimes_{m} \in \\
\|x\| \leq 1}}\left(\sum_{i_{1}, \ldots, i_{m}}\left|x_{i_{1}} \cdots x_{i_{m}}\right|^{2}\right)^{1 / 2} \\
& =\sup _{|\alpha|=m}\left(\left|\lambda_{\alpha}\right| \sqrt{\frac{\alpha !}{m !}}\right) \| \text { id }: \bigotimes_{\pi}^{m} E \longrightarrow \ell_{2}^{n^{m}} \| \\
& =\sup _{|\alpha|=m}\left(\left|\lambda_{\alpha}\right| \sqrt{\frac{\alpha !}{m !}}\right) \| \text { id }: E \longrightarrow \ell_{2}^{n} \|^{m} \text {. }
\end{aligned}
$$

Note that for $N=1$ we again have [10, Corollary 3.2]. Letting $E=\ell_{\infty}^{n}$ and $F=\ell_{p}^{n}$ for some $1 \leq p \leq 2$ we have

$$
\| \text { id }: \ell_{2}^{n} \rightarrow \ell_{p}^{n}\left\|=n^{1 / p-1 / 2},\right\| \text { id }: \ell_{\infty}^{n} \longrightarrow \ell_{2}^{n} \|=n^{1 / 2} .
$$

Also, for every $1 \leq r<\infty$ there exists a constant $C$ so that (see 23, Proposition 45.1])

$$
\int\left\|\sum_{k=1}^{n} g_{k}(\omega) e_{k}\right\|_{\ell_{r}^{n}} d \omega \leq C n^{1 / r}
$$

Corollary 4.3. Let $1 \leq p \leq 2$. For every choice of scalars $\left(\lambda_{\alpha}\right)_{|\alpha|=m}$ there exists $\left(c_{\alpha}\right)_{|\alpha|=m} \subseteq \ell_{p}^{n}$, each $c_{\alpha}$ with entries consisting only of \pm 1 , such that for the $m$ homogeneous polynomial $\sum_{|\alpha|=m} \lambda_{\alpha} c_{\alpha} x^{\alpha}$ we have

$$
\left\|\sum_{|\alpha|=m} \lambda_{\alpha} c_{\alpha} x^{\alpha}\right\|_{\mathcal{P}\left(m \ell_{\infty}^{n}, \ell_{p}^{n}\right)} \leq K \sup _{|\alpha|=m}\left(\left|\lambda_{\alpha}\right| \sqrt{\frac{\alpha !}{m !}}\right) n^{m / 2} n^{1 / p}
$$

where $K>0$ is a constant depending only on $m$.

Proof. From what we just mentioned we have

$$
\int\left\|\sum_{|\alpha|=m} \lambda_{\alpha}\left(\sum_{k=1}^{n} g_{\alpha, k}(\omega) e_{k}\right) x^{\alpha}\right\|_{\mathcal{P}\left(m \ell_{\infty}^{n}, \ell_{p}^{n}\right)} d \omega \leq K \sup _{|\alpha|=m}\left(\left|\lambda_{\alpha}\right| \sqrt{\frac{\alpha !}{m !}}\right) n^{m / 2} n^{1 / p} .
$$

It is a well-known fact that the Bernouilli averages are dominated by the Gaussian averages (see e.g. [14, 12.11]). Hence if $\varepsilon_{\alpha, k}$ are independent Bernouilli random 
variables, then

$$
\begin{aligned}
& \int\left\|\sum_{|\alpha|=m} \lambda_{\alpha}\left(\sum_{k=1}^{n} \varepsilon_{\alpha, k}(\omega) e_{k}\right) x^{\alpha}\right\|_{\mathcal{P}\left(m \ell_{\infty}^{n}, \ell_{p}^{n}\right)} d \omega \\
& \leq \int\left\|\sum_{|\alpha|=m} \lambda_{\alpha}\left(\sum_{k=1}^{n} g_{\alpha, k}(\omega) e_{k}\right) x^{\alpha}\right\|_{\mathcal{P}\left(m \ell_{\infty}^{n}, \ell_{p}^{n}\right)} d \omega \\
& \leq K \sup _{|\alpha|=m}\left(\left|\lambda_{\alpha}\right| \sqrt{\frac{\alpha !}{m !}}\right) n^{m / 2} n^{1 / p} .
\end{aligned}
$$

This means that there exist choices of signs $\varepsilon_{\alpha, k}= \pm 1$ so that

$$
\left\|\sum_{|\alpha|=m} \lambda_{\alpha}\left(\sum_{k=1}^{n} \varepsilon_{\alpha, k} e_{k}\right) x^{\alpha}\right\|_{\mathcal{P}\left(m_{\left.\ell_{\infty}^{n}, \ell_{p}^{n}\right)}\right.} \leq K \sup _{|\alpha|=m}\left(\left|\lambda_{\alpha}\right| \sqrt{\frac{\alpha !}{m !}}\right) n^{m / 2} n^{1 / p} .
$$

Letting $c_{\alpha}=\sum_{k=1}^{n} \varepsilon_{\alpha, k} e_{k} \in E$, the conclusion finally follows.

We can now use the polynomials we just produced to obtain a Dirichlet polynomial whose strip of uniform but not absolute convergence has maximal width. For a fixed $\rho>1$ we have from the preceding corollary polynomials $P_{n} \in \mathcal{P}\left({ }^{m} \ell_{\infty}^{\rho^{n}}, \ell_{p}^{\rho^{n}}\right)$ whose coefficients have entries all equal to \pm 1 satisfying $\left\|P_{n}\right\| \leq K\left(\rho^{n}\right)^{m / 2+1 / p}$, and clearly $\left\|c_{\alpha}\left(P_{n}\right)\right\|_{q}=\left(\rho^{n}\right)^{1 / q}$. By Proposition 4.1 there exists an $m$-homogeneous Dirichlet polynomial $\sum_{\Omega(n)=m} a_{n} / n^{s}$ such that $\sigma_{a}^{\ell_{q}}-\sigma_{u}^{\ell_{p}} \geq \frac{m-2(1 / p-1 / q)}{2 m}$. On the other hand, we already know $\sigma_{a}^{\ell_{q}}-\sigma_{u}^{\ell_{p}} \leq T_{m}(p, q) \leq \frac{m-2(1 / p-1 / q)}{2 m}$, and this gives

$$
\sigma_{a}^{\ell_{q}}\left(\sum a_{n} / n^{s}\right)-\sigma_{u}^{\ell_{p}}\left(\sum a_{n} / n^{s}\right)=\frac{m-2(1 / p-1 / q)}{2 m} .
$$

4.2. The case $1 \leq p \leq 2 \leq q$. In this second case we use a totally different technique following [4, Section 3]. Let us first note that if $P \in \mathcal{P}\left({ }^{m} c_{0}, \ell_{p}\right)$ is such that $\inf \left\|c_{\alpha}(P)\right\|_{\infty} \geq K\left(\rho^{n}\right)^{\beta}$, we automatically have the same inequality for $\inf \left\|c_{\alpha}(P)\right\|_{q}$. It is therefore enough to obtain polynomials that satisfy the desired inequality for $\|\cdot\|_{\infty}$.

Let us choose a prime number $\rho>m$ and consider the $\rho \times \rho$ matrix $M_{1}=\left(m_{r s}\right)_{r, s}$ with $m_{r s}=e^{2 \pi i(r-s) / \rho}$. Starting from this we define matrices

$$
M_{2}=\left(\begin{array}{ccc}
m_{11} M_{1} & \cdots & m_{1 \rho} M_{1} \\
\vdots & & \vdots \\
m_{\rho 1} M_{1} & \cdots & m_{\rho \rho} M_{1}
\end{array}\right), \ldots, M_{n}=\left(\begin{array}{ccc}
m_{11} M_{n-1} & \cdots & m_{1 \rho} M_{n-1} \\
\vdots & & \vdots \\
m_{\rho 1} M_{n-1} & \cdots & m_{\rho \rho} M_{n-1}
\end{array}\right) .
$$

Note that each $M_{j}$ is a $\rho^{j} \times \rho^{j}$ matrix. It is easily seen that $M_{n}=\left(a_{r s}\right)_{r, s=1, \ldots, \rho^{n}}$ satisfies first that $\sum_{t} a_{r t} \bar{a}_{s t}=\rho^{n} \delta_{r s}$, second that $\left|a_{r s}\right|=1$ and third that $a_{r s}^{\rho}=1$. We then consider the $m$-linear mapping $A_{n} \in \mathcal{L}\left({ }^{m} \ell_{\infty}^{\rho^{n}}, \ell_{p}^{\rho^{n}}\right)$ defined by $A_{n}\left(e_{i_{1}}, \ldots, e_{i_{m}}\right)$ $=\left(\xi_{k}\right)_{k}$ with $\xi_{k}=a_{i_{1} i_{2}} \cdots a_{i_{m-1} i_{m}} \delta_{i_{m} k}$. We know from [13, Section 3.2] that this mapping satisfies $\left\|A_{n}\right\| \leq\left(\rho^{n}\right)^{m / 2}\left(\rho^{n}\right)^{1 / p-1 / 2}$. We symmetrize it and consider the associated $m$-homogeneous polynomial $P_{n}(z)=\sum_{|\alpha|=m} c_{\alpha}^{(n)} z^{\alpha}$ whose coefficients are given by

$$
c_{\alpha}^{(n)}=\frac{1}{\alpha !} \sum_{\sigma \in \Sigma_{m}} A_{n}\left(e_{i_{\sigma(1)}}, \ldots, e_{i_{\sigma(m)}}\right)
$$


where for each $\boldsymbol{i}=\left(i_{1}, \ldots, i_{m}\right)$ the corresponding $\alpha$ is defined by $\alpha_{r}=\left|\left\{k: i_{k}=r\right\}\right|$ (i.e. $\alpha_{1}$ is the number of 1's in $\boldsymbol{i}, \alpha_{2}$ is the number of 2's, ..) and $\Sigma_{m}$ is the group of permutations of $\{1, \ldots, m\}$. By the very definition of $A_{n}$ each $A_{n}\left(e_{j_{1}}, \ldots, e_{j_{m}}\right)$ is a vector in $\ell_{p}^{\rho^{n}}$ whose entries are all 0 except for the $j_{m}$-th one, in which there is the value $a_{j_{1} j_{2}} \cdots a_{j_{m-1} j_{m}}$; hence the entries of $\alpha ! c_{\alpha}^{(n)} \in \ell_{p}^{\rho^{n}}$ are all sums of vectors of this kind. More precisely, if $\Sigma_{m, k}=\left\{\sigma \in \Sigma_{m}: i_{\sigma(m)}=k\right\}$, for $k=1, \ldots, \rho^{n}$, then

$$
\alpha ! c_{\alpha}^{(n)}(k)=\sum_{\sigma \in \Sigma_{m, k}} a_{i_{\sigma(1)} i_{\sigma(2)}} \cdots a_{i_{\sigma(m-1)} i_{\sigma(m)}} .
$$

Each $a_{i_{\sigma(1)} i_{\sigma(2)}} \cdots a_{i_{\sigma(m-1)} i_{\sigma(m)}}$ is a $\rho$-th root of unity; hence if we put $\xi=e^{2 \pi i / \rho}$ there is some $j_{0}$ such that $a_{i_{\sigma(1)} i_{\sigma(2)}} \cdots a_{i_{\sigma(m-1)} i_{\sigma(m)}}=\xi^{j_{0}}$. Then $\alpha ! c_{\alpha}^{(n)}(k)$ is a sum of elements of this form and we can write $\alpha ! c_{\alpha}^{(n)}(k)=\sum_{j=0}^{\rho-1} \lambda_{j}^{(k)} \xi^{j}$, where each $\lambda_{j}^{(k)}$ is just the number of times that the $\xi^{j}$ is repeated in the sum of $\alpha ! c_{\alpha}^{(n)}(k)$. Then the sum of all the $\lambda_{j}^{(k)}$ 's is the total number of terms in the sum, i.e. $\sum_{j=0}^{\rho-1} \lambda_{j}^{(k)}=$ $\left|\Sigma_{m, k}\right| \leq\left|\Sigma_{m}\right|=m !$. Just as before we can write

$$
\begin{aligned}
\alpha ! \sum_{k} c_{\alpha}^{(n)}(k)=\sum_{k} \sum_{\sigma \in \Sigma_{m, k}} a_{i_{\sigma(1)} i_{\sigma(2)}} \cdots a_{i_{\sigma(m-1)} i_{\sigma(m)}} & \\
=\sum_{\sigma \in \Sigma_{m}} a_{i_{\sigma(1)} i_{\sigma(2)}} \cdots a_{i_{\sigma(m-1)} i_{\sigma(m)}} & =\sum_{j=0}^{\rho-1} \lambda_{j} \xi^{j} .
\end{aligned}
$$

Again, each $\lambda_{j} \in \mathbb{N}$ is the number of times that the term $\xi^{j}$ appears in the sum. Let us now show that the last term cannot equal 0 . If this were the case we would have (recall that the sum of all the $\rho$-th roots of unity equals 0 )

$$
0=-\lambda_{0} \sum_{j=1}^{\rho-1} \xi^{j}+\sum_{j=1}^{\rho-1} \lambda_{j} \xi^{j}=\sum_{j=1}^{\rho-1}\left(\lambda_{j}-\lambda_{0}\right) \xi^{j} .
$$

Since $\left\{\xi, \xi^{2}, \ldots, \xi^{\rho-1}\right\}$ is linearly independent over $\mathbb{Q}$, this would imply $\lambda_{0}=\lambda_{1}=$ $\cdots=\lambda_{\rho-1}=\lambda$. But then $m !=\sum_{j=0}^{\rho-1} \lambda_{j}=\rho \lambda$ and, because $\lambda \in \mathbb{N}$, this contradicts the fact that $\rho$ is prime and $>m$. Hence

$$
0<\left|\sum_{k} c_{\alpha}^{(n)}(k)\right| \leq \sum_{k}\left|c_{\alpha}^{(n)}(k)\right| .
$$

From this we have $\left\|c_{\alpha}^{(n)}\right\|_{\infty}>0$ for all $\alpha$ and all $n$. On the other hand, the fact that $\sum_{j=0}^{\rho-1} \lambda_{j}^{(k)} \leq m$ ! gives that there is only a finite number of possible values for $c_{\alpha}^{(n)}(k)$ (since there is only a finite number of $\rho$-tuples of natural numbers that sum up to $m$ !, and then just a finite number of possible sums $\left.\sum_{j=0}^{\rho-1} \lambda_{j}^{(k)} \xi^{j}\right)$; hence $\inf \left\{\left\|c_{\alpha}^{(n)}\right\|_{\infty}: \alpha \in \mathbb{N}_{0}^{n}, n \in \mathbb{N}\right\}>0$ and

$$
\left\|P_{n}\right\| \leq\left\|A_{n}\right\| \leq\left(\rho^{n}\right)^{m / 2}\left(\rho^{n}\right)^{1 / p-1 / 2} .
$$

This gives $\gamma=m / 2+1 / p-1 / 2$ and $\beta=0$ in Proposition 4.1. Hence there exists an $m$-homogeneous Dirichlet polynomial $\sum_{\Omega(n)=m} a_{n} / n^{s}$ that satisfies

$$
\sigma_{a}^{\ell_{q}}-\sigma_{u}^{\ell_{p}}=\frac{m-2(1 / p-1 / 2)}{2 m} .
$$


4.3. The case $2 \leq p$. Let us consider the $m$-homogeneous Dirichlet polynomial $\sum_{\Omega(n)=m} e_{n} / n^{s}$. We know that $\sigma_{a}^{\ell_{q}}-\sigma_{u}^{\ell_{p}} \leq T_{m}(p, q) \leq 1 / p^{\prime}$. It is a well-known fact that the scalar valued Dirichlet series $\sum 1 / n^{s}$ (the Riemann $\zeta$-function) satisfies $\sigma_{u}=\sigma_{a}=1$. Using this fact it is not difficult to prove that this is also the case of the corresponding $m$-homogeneous Dirichlet polynomial $\sum_{\Omega(n)=m} 1 / n^{s}$. It is then clear that $\sigma_{a}\left(\sum_{\Omega(n)=m} e_{n} / n^{s}\right)=1$. On the other hand we have

$$
\sup _{t \in \mathbb{R}}\left\|\sum_{\substack{n=1 \\ \Omega(n)=m}}^{N} e_{n} n^{i t}\right\|_{p}=\sup _{t \in \mathbb{R}}\left(\sum_{\substack{n=1 \\ \Omega(n)=m}}^{N}\left|n^{i t}\right|^{p}\right)^{1 / p} \leq N^{1 / p} .
$$

Using (8) we obtain $\sigma_{u}^{\ell_{p}} \leq 1 / p$. This gives $\sigma_{a}^{\ell_{q}}-\sigma_{u}^{\ell_{p}} \geq 1-1 / p=1 / p^{\prime}$ and finishes the proof of (1).

\section{The Proof: Part III. The intermediate widths}

In order to complete the proof of Theorem 1.1 we still have to show that there are $m$-homogeneous Dirichlet polynomials that attain any intermediate given width of uniform but not absolute convergence. Adapting an idea of Bohr 44, page 622] we now show that, once we have attained some width with a Dirichlet series, any smaller width can also be attained with a perturbation of the original Dirichlet series.

If $\sum a_{n} / n^{s}$ and $\sum b_{n} / n^{s}$ are two Dirichlet series in a Banach space $X$ for which $\sigma_{a}\left(\sum a_{n} / n^{s}\right) \neq \sigma_{a}\left(\sum b_{n} / n^{s}\right)$, then

$$
\sigma_{a}\left(\sum\left(a_{n}+b_{n}\right) / n^{s}\right)=\max \left(\sigma_{a}\left(\sum a_{n} / n^{s}\right), \sigma_{a}\left(\sum b_{n} / n^{s}\right)\right) .
$$

Indeed, this is implied by the fact that a sum of two series cannot be an absolutely convergent series if one is absolutely convergent and the other is not. Also, if $\sigma_{u}\left(\sum a_{n} / n^{s}\right) \neq \sigma_{u}\left(\sum b_{n} / n^{s}\right)$, then

$$
\sigma_{u}\left(\sum\left(a_{n}+b_{n}\right) / n^{s}\right)=\max \left(\sigma_{u}\left(\sum a_{n} / n^{s}\right), \sigma_{u}\left(\sum b_{n} / n^{s}\right)\right) .
$$

We again take a general approach, using operators.

Lemma 5.1. Let $v: X \rightarrow Y$ be a non-zero operator, $\sum a_{n} / n^{s}$ a Dirichlet series in a Banach space $X$ and $x_{0} \in X$ such that $v x_{0} \neq 0$. Let $\sigma_{a}^{Y}$ be the abscissa of absolute convergence of $\sum v a_{n} / n^{s}$ and $\sigma_{u}^{X}$ be the abscissa of uniform convergence of $\sum a_{n} / n^{s}$. Then for each $0<\sigma<\sigma_{a}^{Y}-\sigma_{u}^{X}$ the Dirichlet series

$$
\sum_{n} b_{n} \frac{1}{n^{s}}=\sum_{n} a_{n} \frac{1}{n^{s}}+\sum_{n} \frac{x_{0}}{n^{1-\sigma_{a}^{Y}+\sigma}} \frac{1}{n^{s}}
$$

satisfies

$$
\sigma_{a}\left(\sum v b_{n} / n^{s}\right)-\sigma_{u}\left(\sum b_{n} / n^{s}\right)=\sigma .
$$

If moreover $\sum a_{n} / n^{s}$ is an m-homogeneous Dirichlet polynomial, then

$$
\sum_{\Omega(n)=m} b_{n} \frac{1}{n^{s}}=\sum_{\Omega(n)=m} a_{n} \frac{1}{n^{s}}+\sum_{\Omega(n)=m} \frac{x_{0}}{n^{1-\sigma_{a}^{Y}+\sigma}} \frac{1}{n^{s}}
$$

defines an $m$-homogenous Dirichlet polynomial for which we have

$$
\sigma_{a}\left(\sum v b_{n} / n^{s}\right)-\sigma_{u}\left(\sum b_{n} / n^{s}\right)=\sigma .
$$


Proof. It is easily seen, using the fact that the Riemann $\zeta$-function and its corresponding $m$-homogeneous polynomials have abscissas of absolute and of uniform convergence equal to 1 , that

$$
\sigma_{a}\left(\sum \frac{v x_{0}}{n^{1-\sigma_{a}^{Y}+\sigma}} \frac{1}{n^{s}}\right)=\sigma_{u}\left(\sum \frac{x_{0}}{n^{1-\sigma_{a}^{Y}+\sigma}} \frac{1}{n^{s}}\right)=\sigma_{a}^{Y}-\sigma
$$

(the same for the corresponding polynomials). This, together with (14), gives $\sigma_{a}\left(\sum v b_{n} / n^{s}\right)=\sigma_{a}^{Y}$. On the other hand, since $\sigma_{a}^{Y}-\sigma>\sigma_{a}^{Y}-\sigma_{a}^{Y}+\sigma_{u}^{X}=\sigma_{u}^{X}$, we have from (15) that $\sigma_{u}\left(\sum b_{n} / n^{s}\right)=\sigma_{a}^{Y}-\sigma$. This clearly gives the conclusion.

The preceding lemma applied to $v=\mathrm{id}: \ell_{p} \hookrightarrow \ell_{q}$ and the examples obtained in Section 5 finally complete the proof of Theorem 1.1.

\section{The OPERATOR POINT OF VIEW}

Let us now come back to our original question about why for finite dimensional spaces the maximal width of uniform but not absolute convergence for homogeneous Dirichlet polynomials heavily depends on the degree of the polynomial, but for infinite dimensional spaces this dependence disappears. Our aim was to analyze and, if possible, understand this phenomenon. We have seen in Theorem 1.1 that if for $\sum a_{n} / n^{s}$ in $\ell_{p}$ we take the abscissa of absolute convergence in a larger $\ell_{q}$, then again the dependence on the degree appears. Let us point out that if we take $p=q$ in Theorem 1.1 we again have (4) and that $T_{m}(p, q)=\frac{m-1}{2 m}$ if and only if $p=1$ and $q \geq 2$. We now slightly change our point of view in hopes that this will help bring more light to our original question of the 'invisible polynomials'. We now want to look at the problem from the point of view of operators, inserting $T_{m}(p, q)$ in a more general framework.

Let $v$ be a non-zero operator between Banach spaces $X$ and $Y$. If $\sum_{n} a_{n} \frac{1}{n^{s}}$ is a Dirichlet series in $X$ with abscissa of uniform convergence $\sigma_{u}$, then $\sum_{n} a_{n} \frac{1}{n^{\sigma_{u}+T(X)+\varepsilon}}$ by definition converges absolutely, but depending on the operator $v$ the half plane on which the series $\sum_{n} v\left(a_{n}\right) \frac{1}{n^{s}}$ converges absolutely might be very different. Our main aim now is to look at the number

$$
T_{m}(v):=\sup \sigma_{a}^{Y}-\sigma_{u}^{X},
$$

where the sup is taken with respect to all $m$-homogeneous Dirichlet polynomials $\sum_{n} a_{n} \frac{1}{n^{s}}$ in $X$, the abscissa $\sigma_{u}^{X}$ of uniform convergence of $\sum_{n} a_{n} \frac{1}{n^{s}}$ is taken in $X$, but the abscissa $\sigma_{a}^{Y}$ of absolute convergence is taken with respect to the Dirichlet series $\sum_{n} v\left(a_{n}\right) \frac{1}{n^{s}}$ in $Y$. Note that $T_{m}(v)$ can also be seen as the infimum over all $r>0$ so that if $\sum_{\Omega(n)=m} a_{n} \frac{1}{n^{s}}$ converges uniformly in $[\operatorname{Re} s \geq \sigma]$, then $\sum_{\Omega(n)=m} v\left(a_{n}\right) \frac{1}{n^{s}}$ converges absolutely in $[\operatorname{Re} s \geq \sigma+r+\varepsilon]$ for every $\varepsilon>0$. Somehow $T_{m}(v)$ gives an idea of how much the operator $v$ improves (or at least modifies) the summability of $m$-homogeneous Dirichlet polynomials. With this notation, (3) and its counterpart for finite dimensional $X$ can be rewritten using the notation $T_{m}\left(\mathrm{id}_{\mathbb{C}}\right)$ and $T_{m}\left(\mathrm{id}_{X}\right)$.

Clearly for each $v$ the sequence $\left(T_{m}(v)\right)_{m}$ is non-increasing and

$$
T_{m}(v) \geq T_{m}\left(\operatorname{id}_{\mathbb{C}}\right)=\frac{m-1}{2 m} .
$$


The situation exposed in [11] (that gave rise to our problem) can now be rewritten as

$$
T_{m}(X)=T_{m}\left(\operatorname{id}_{X}\right)= \begin{cases}\frac{m-1}{2 m} & \text { if } \operatorname{dim} X<\infty, \\ \frac{1}{\cot (X)^{\prime}} & \text { if } \operatorname{dim} X=\infty,\end{cases}
$$

and the question was to try to analyze why there is this dramatic difference between the finite and the infinite dimensional case.

It is also clear that $T_{m}(p, q)=T_{m}\left(\mathrm{id}: \ell_{p} \hookrightarrow \ell_{q}\right)$. Following ideas similar to those used in Section 3 we can also give a general upper bound for $T_{m}(v)$. Let us recall (see e.g. 14, Chapter 10]) that an operator $v: X \rightarrow Y$ between Banach spaces is called $(r, 1)$-summing if there exists a constant $C>0$ so that for every $A \in \mathcal{L}\left(c_{0}, X\right)$

$$
\left(\sum_{i=1}^{\infty}\left\|v A\left(e_{i}\right)\right\|^{r}\right)^{1 / r} \leq C\|A\| .
$$

Operators that are $(1,1)$-summing are simply called summing. As a natural generalization of this and inspired by [4, Theorem I], $(r, 1)$-summing operators of order $m$ are defined in [13] as those $v: X \rightarrow Y$ for which there exists $C>0$ such that for every $A \in \mathcal{L}\left({ }^{m} c_{0}, X\right)$

$$
\left(\sum_{i_{1}, \ldots, i_{m}=1}^{\infty}\left\|v A\left(e_{i_{1}}, \ldots, e_{i_{m}}\right)\right\|^{r}\right)^{1 / r} \leq C\|A\| .
$$

Then $B H_{m}(v)$, the $m$-th Bohnenblust-Hille index of $v$, is defined as the infimum over all $r$ so that $v$ is $(r, 1)$-summing of order $m$. Clearly, $B H_{1}(v)$ is the infimum over the $r$ 's so that $v$ is $(r, 1)$-summing.

With this notation [4, Theorem I] reads as $B H_{m}\left(\operatorname{id}_{\mathbb{C}}\right)=\frac{2 m}{m+1}$, and this is used to obtain upper bounds for $T$ (the original, scalar one). Just as in the classical case, so also in this setting the Bohnenblust-Hille indices help in giving upper bounds for $T_{m}(v)$. We even get equality in the case $m=1$; it remains an open problem if equality can be achieved in the general case.

Proposition 6.1. For any operator $0 \neq v: X \rightarrow Y$ we have

$$
\frac{1}{B H_{1}(v)^{\prime}}=T_{1}(v) \leq T_{m}(v) \leq \frac{1}{B H_{m}(v)^{\prime}} .
$$

Proof. The proof of the last inequality follows exactly the same steps as in Section 3. using (12). To prove the first equality, let us choose $r>T_{1}(v)$ and show that $r \geq 1 / B H_{1}(v)^{\prime}$. If $q=1 / r$ this is equivalent to $q^{\prime} \geq B H_{1}(v)$. We then take $A \in \mathcal{L}\left(c_{0}, X\right)$ and $c_{n}:=A\left(e_{n}\right)$. Let us show in a first step that $\sum_{n}\left\|v c_{n} x_{n}\right\|$ is finite for every $x \in \ell_{q}$. We take $x \in \ell_{q}$ and $\sigma$ a permutation so that $\left(\left|x_{\sigma(n)}\right|\right)_{n}$ is the decreasing rearrangement of $x$. Then $\sup _{n}\left|x_{\sigma(n)}\right| n^{1 / q}=K<\infty$. On the other hand, by the prime number theorem there exists $C>0$ such that $p_{n} \leq C n \log n$ for all $n$. Then for $\varepsilon:=\frac{r}{T_{1}(v)}-1>0$ there exists $D>0$ so that $\frac{1}{n} \leq D \frac{1}{p_{n}^{1 /(1+\varepsilon)}}$, and hence for every $n$,

$$
\left|x_{\sigma(n)}\right| \leq K \frac{1}{n^{\frac{1}{q}}} \leq K D^{r} \frac{1}{p_{n}^{\frac{r}{1+\varepsilon}}} .
$$

We now consider the 1-homogeneous Dirichlet polynomial

$$
\sum_{n} c_{\sigma(n)} \frac{1}{p_{n}^{s}}
$$


Clearly, $\left(c_{n}\right)_{n}$ is weakly 1-summable (i.e., $\left(x^{\prime}\left(c_{n}\right)\right)_{n}$ is summable for each $\left.x^{\prime} \in X\right)$, which implies that $\tilde{A}\left(e_{n}\right):=c_{\sigma(n)}$ defines a bounded, linear operator from $c_{0}$ to $X$ (see e.g. [14, Theorem 1.9]) and, by (10), the Dirichlet polynomial has an abscissa of uniform convergence $\sigma_{u}^{X} \leq 0$. Since $\frac{r}{1+\varepsilon}>T_{1}(v) \geq \sigma_{a}^{Y}-\sigma_{u}^{X} \geq \sigma_{a}^{Y}$, we see that

$$
\sum_{n}\left\|v c_{\sigma(n)} x_{\sigma(n)}\right\|=\sum_{n}\left\|v c_{\sigma(n)}\right\|\left|x_{\sigma(n)}\right| \leq K D^{r} \sum_{n}\left\|v c_{\sigma(n)}\right\| \frac{1}{p_{n}^{\frac{r}{1+\varepsilon}}}<\infty .
$$

Altogether we have shown that $\sum_{n}\left\|v c_{n} x_{n}\right\|=\sum_{n}\left\|v c_{\sigma(n)} x_{\sigma(n)}\right\|<\infty$ for every $x \in \ell_{q}$. By Hölder's inequality, $\left(\left\|v c_{n}\right\|\right)_{n} \in \ell_{q^{\prime}}$ and the mapping $\mathcal{L}\left(c_{0}, X\right) \rightarrow \ell_{q^{\prime}}(Y)$ (the space of $q^{\prime}$-summable sequences in $Y$ ) that maps $A$ to $\left(v c_{n}\right)_{n}$ is well defined, and a simple closed-graph argument shows that it is continuous. This gives $q^{\prime} \geq B H_{1}(v)$ and completes the proof.

Theorem 6.2. Let $v$ be an operator with values in a cotype 2 space and $m \in \mathbb{N}$. Then

(a) $\max \left\{\frac{m-1}{2 m}, T_{1}(v)\right\} \leq T_{m}(v) \leq \frac{m-1}{2 m}+\frac{T_{1}(v)}{m}$.

(b) $v$ is $(1+\varepsilon, 1)$-summing for every $\varepsilon>0$ if and only if

$$
T_{m}(v)=\frac{m-1}{2 m}
$$

holds for every $m$. In particular, this equality holds for any summing operator.

Proof. The lower bound in (a) follows from (16) and the fact that $\left(T_{m}(v)\right)_{m}$ forms an increasing sequence. For the upper bound it is known from [13, Lemma 3] that if $v$ takes values in a cotype 2 space, then

$$
B H_{m}(v) \leq \frac{2 m}{m+2\left(\frac{1}{B H_{1}(v)}-\frac{1}{2}\right)} .
$$

An easy computation now gives the estimation.

For part (b), if $v$ is $(1+\varepsilon, 1)$-summing for every $\varepsilon>0$, then $B H_{1}(v)=1$; hence by the preceding result $T_{1}(v)=0$, and then $T_{m}(v)=\frac{m-1}{2 m}$ by (a). For the converse, if the equality holds for every $m$, in particular we have $T_{1}(v)=0$. By Proposition 6.1 this implies $B H_{1}(v)=1$ and gives the conclusion. For the final remark recall that every summing operator factors through a Hilbert space (see [14, Theorem 2.8, Theorem 2.13]).

By [19, (1.1)] (see also [14, p. 208]), every operator $v: \ell_{1} \rightarrow \ell_{p}$ with $1 \leq p<\infty$ is $(r, 1)$-summing for $1 / r=1-|(1 / p)-(1 / 2)|$. Then using the preceding theorem and Proposition 6.1 we get

Corollary 6.3. Let $v: \ell_{1} \rightarrow \ell_{p}$ with $1 \leq p \leq 2$. Then

$$
T_{m}(v) \leq \frac{m-2+\frac{2}{p}}{2 m} .
$$

Let us finally go back to our original problem (re)formulated in (17); we reprove (17) in a systematic way that we feel helps in clarifying the subject. At this stage it is clear that the maximal width of the corresponding strip for a given operator is very much related to its summability properties (see e.g. Proposition 6.1). 
By the Dvoretzky-Rogers Theorem (see e.g. 14, Theorem 2.18]), the identity of a Banach space is summing if and only if it is finite dimensional. Then Theorem 6.2 explains why in (17) only the finite dimensional spaces have precisely $T\left(\mathrm{id}_{X}\right)=$ $\frac{m-1}{2 m}$.

Again by the Dvoretzky-Rogers Theorem (see e.g. [14, Theorem 10.5]) the identity on an infinite dimensional Banach space is not $(r, 1)$-summing for any $1 \leq r<2$. Hence it is now no surprise that $\cot (X)$ appears, since by [21, Théorème 1.1] (see also [14, page 304]) this is exactly the infimum over all $r$ so that $\operatorname{id}_{X}$ is $(r, 1)$ summing (then the 'best summability' we can expect from $\mathrm{id}_{X}$ ); in other words $B H_{1}\left(\operatorname{id}_{X}\right)=\cot (X)$. From [7, Theorem 3.2] we have $B H_{m}\left(\operatorname{id}_{X}\right) \leq \cot (X)$ for every infinite dimensional Banach space $X$ (even equality by [13, Proposition 7]). With Proposition 6.1 this altogether yields that for infinite dimensional Banach spaces,

$$
\frac{1}{\cot (X)^{\prime}}=\frac{1}{B H_{1}\left(\operatorname{id}_{X}\right)^{\prime}}=T_{1}\left(\operatorname{id}_{X}\right) \leq T_{m}\left(\operatorname{id}_{X}\right) \leq \frac{1}{B H_{m}\left(\operatorname{id}_{X}\right)^{\prime}}=\frac{1}{\cot (X)^{\prime}} .
$$

\section{ACKNOWLEDGEMENTS}

We wish to thank the referee for his/her careful reading and the valuable suggestions that helped improve the paper.

\section{REFERENCES}

[1] T. M. Apostol. Introduction to analytic number theory. Springer-Verlag, New York, 1976. Undergraduate Texts in Mathematics. MR0434929 (55:7892)

[2] T. M. Apostol. Modular functions and Dirichlet series in number theory, volume 41 of Graduate Texts in Mathematics. Springer-Verlag, New York, second edition, 1990. MR.1027834 (90j:11001)

[3] H. P. Boas. The football player and the infinite series. Notices Amer. Math. Soc., 44(11):14301435, 1997. MR.1488569 (99a:11101)

[4] H. F. Bohnenblust and E. Hille. On the absolute convergence of Dirichlet series. Ann. of Math. (2), 32(3):600-622, 1931. MR1503020

[5] H. Bohr. Über die Bedeutung der Potenzreihen unendlich vieler Variabeln in der Theorie der Dirichlet-schen Reihen $\sum \frac{a_{n}}{n^{2}}$. Nachr. Ges. Wiss. Göttingen, Math. Phys. Kl., pages 441-488, 1913.

[6] H. Bohr. Über die gleichmäßige Konverenz Dirichletscher Reihen. J. Reine Angew. Math., 143:203-211, 1913.

[7] F. Bombal, D. Pérez-García, and I. Villanueva. Multilinear extensions of Grothendieck's theorem. Q. J. Math., 55(4):441-450, 2004. MR2104683 (2005i:47032)

[8] A. Defant, J. C. Díaz, D. García, and M. Maestre. Unconditional basis and Gordon-Lewis constants for spaces of polynomials. J. Funct. Anal., 181(1):119-145, 2001. MR:1818112 (2002b:46030)

[9] A. Defant and K. Floret. Tensor norms and operator ideals, volume 176 of NorthHolland Mathematics Studies. North-Holland Publishing Co., Amsterdam, 1993. MR.1209438 (94e:46130)

[10] A. Defant, D. García, and M. Maestre. Bohr's power series theorem and local Banach space theory. J. Reine Angew. Math., 557:173-197, 2003. MR1978407 (2004d:46012)

[11] A. Defant, D. García, M. Maestre, and D. Pérez-García. Bohr's strip for vector valued Dirichlet series. Math. Ann., 342:533-551, 2008. MR.2430989

[12] A. Defant, M. Maestre, and C. Prengel. Domains of convergence for monomial expansions of holomorphic functions in infinitely many variables. J. Reine Angew. Math., 634:13-49, 2009. MR2560405

[13] A. Defant and P. Sevilla-Peris. A new multilinear insight on the classical Littlewood's 4/3 inequality. J. Funct. Anal., 256(5):1642-1664, 2009. MR2490233 
[14] J. Diestel, H. Jarchow, and A. Tonge. Absolutely summing operators, volume 43 of Cambridge Studies in Advanced Mathematics. Cambridge University Press, Cambridge, 1995. MR.1342297 (96i:46001)

[15] S. Dineen. Complex analysis on infinite-dimensional spaces. Springer Monographs in Mathematics. Springer-Verlag London Ltd., London, 1999. MR1705327 (2001a:46043)

[16] K. Floret. Natural norms on symmetric tensor products of normed spaces. Note Mat., 17:153188 (1999), 1997. MR 1749787 (2001g:46038)

[17] H. Hedenmalm. Dirichlet series and functional analysis. In The legacy of Niels Henrik Abel, pages 673-684. Springer, Berlin, 2004. MR2077590 (2005h:30002)

[18] S. V. Konyagin and H. Queffélec. The translation $\frac{1}{2}$ in the theory of Dirichlet series. Real Anal. Exchange, 27(1):155-175, 2001/02. MR.1887689 (2002k:42012)

[19] S. Kwapień. Some remarks on $(p, q)$-absolutely summing operators in $l_{p}$-spaces. Studia Math., 29:327-337, 1968. MR0231212 (37:6767)

[20] J. Lindenstrauss and L. Tzafriri. Classical Banach spaces. II, volume 97 of Ergebnisse der Mathematik und ihrer Grenzgebiete [Results in Mathematics and Related Areas]. SpringerVerlag, Berlin, 1979. Function spaces. MR.540367(81c:46001)

[21] B. Maurey and G. Pisier. Séries de variables aléatoires vectorielles indépendantes et propriétés géométriques des espaces de Banach. Studia Math., 58(1):45-90, 1976. MR0443015 (56:1388)

[22] H. Queffélec. H. Bohr's vision of ordinary Dirichlet series; old and new results. J. Anal., 3:43-60, 1995. MR.1340125 (97a:11137)

[23] N. Tomczak-Jaegermann. Banach-Mazur distances and finite-dimensional operator ideals, volume 38 of Pitman Monographs and Surveys in Pure and Applied Mathematics. Longman Scientific \& Technical, Harlow, 1989.

Institute of Mathematics, Carl von Ossietzky University, D-26111 Oldenburg, GerMANY

E-mail address: defant@mathematik.uni-oldenburg.de

Institute of Mathematics, Carl von Ossietzky University, D-26111 Oldenburg, Germany - And - Departamento de Matemática Aplicada and IUMPA, ETSMRE, Universidad Politécnica de Valencia, Av. Blasco Ibáñez, 21, E-46010 Valencia, Spain

E-mail address: psevilla@mat.upv.es 